\title{
Resistencia primaria a fármacos anti-tuberculosos en Chile 2011-2012
}

Fabiola Arias, Angélica Scappaticcio y Tania Herrera

Instituto de Salud Pública de Chile. Sección Micobacterias (FA, AS). Ministerio de Salud de Chile. Directora del Programa de Control y Eliminación de la Tuberculosis (TH).

Recibido: 30 de junio de 2015 Aceptado: 7 de julio de 2015

Correspondencia a: Tania Herrera Martínez tania.herrera@minsal.cl

\section{Primary resistance to anti tuberculosis drugs in Chile 2011-2012}

This publication presents the results of the Chilean initial study of resistance to first line anti-tuberculous drugs. The study was carried out between 2011 and 2012 by the National Reference Laboratory of the Institute of Public Health, as part of the Drug Surveillance Resistance in tuberculosis (TB) promoted by the World Health Organization. Methodology: Cross-sectional study performed using cluster sampling, representative of the entire country as recommended by the World Health Organization. Susceptibility testing to isoniazid, rifampicin, streptomycin, ethambutol and pyrazinamide was performed through the proportion method or Wayne's enzymatic method, as appropriate. Results: 594 susceptibility tests were performed, showing an overall level of TB drug resistance of $8.6 \%$ and a prevalence of multidrug resistance of $1.3 \%$. Indeed, the study showed a decrease in streptomycin resistance and an increase of isoniazid resistance in both mono-resistance and accumulated resistance compared to previous studies. No cases of mono-resistance to rifampicin were detected. Conclusion: An increased resistance to anti-TB drugs in Chile is observed, which despite being still low, is no less worrisome. Since 2014 the monitoring of drug resistance to TB is universally performed to avoid sub - diagnosis and treat each case according to the susceptibility profile.

Key words: Tuberculosis, initial resistance, Chile.

Palabras clave: Tuberculosis, resistencia inicial, Chile.

L a tuberculosis $(\mathrm{TBC})$ resistente a fármacos es un grave problema de salud pública en muchos países y una amenaza para el control de la TBC en el mundo. Para el año 2013, la Organización Mundial de la Salud (OMS) estimó la existencia de 480.000 casos nuevos de TBC multidrogorresistente (TBC-MDR) y aproximadamente 210.000 muertes por esta causa. Según los datos del último reporte global de TBC, en el mundo, $3,5 \%$ de los casos nuevos y $20,5 \%$ de los casos previamente tratados tienen TBC-MDR ${ }^{1}$.

En 1994 la OMS lanzó el Proyecto Mundial de Vigilancia de la Fármaco-resistencia a los Antituberculosos, el que actualmente recolecta y analiza la información de 144 países que realizan una vigilancia sistemática de la susceptibilidad a fármacos. La forma más común de realizar esta vigilancia en aquellos países que no cuentan con estudios de susceptibilidad rutinarios para todos los pacientes con TBC es a través de estudios especiales llevados a cabo cada 3 a 5 años. Sin embargo, varios de los países con alta carga de TBC o de TBC-MDR sólo recolectan datos a nivel sub-nacional (Brasil, India y la Federación Rusa, entre otros), por lo que aún falta por avanzar en este ámbito ${ }^{1}$.

En Chile, el laboratorio de referencia de TBC del Instituto de Salud Pública (ISP) realiza la vigilancia para Mycobacterium tuberculosis desde el año 1961, conduciendo estudios de resistencia primaria (casos nuevos) periódicamente desde 1971 y vigilando la resistencia adquirida en forma rutinaria a todos los casos antes tratados $^{2}$. Esta publicación muestra los resultados del último estudio de resistencia inicial realizado entre los años 2011 y 2012. Ésta es la última ocasión en que se llevará a cabo un estudio de este tipo, ya que a partir del año 2014 el Programa de Tuberculosis ha ampliado la vigilancia de la resistencia a fármacos anti-tuberculosos a todos los casos pulmonares nuevos y antes tratados con confirmación bacteriológica, lo que representa un avance sustantivo para el manejo de estos pacientes ${ }^{3}$.

\section{Metodología}

Para la realización de este estudio transversal de resistencia inicial se usó la técnica de muestreo por conglomerados recomendada por la $\mathrm{OMS}^{4}$, basada en recolectar muestras pulmonares (esputo o lavado bronco-alveolar) representativas de todos los Servicios de Salud del país según su propia incidencia.

El tamaño de la muestra se determinó en base al número total de casos nuevos pulmonares con bacteriología positiva del año anterior en el país (baciloscopia y cultivo) y la proporción esperada de resistencia a rifampicina según los datos disponibles (precisión 1\%; intervalo de confianza 95\%). El tamaño de la muestra calculado se 
aumentó en $10 \%$ por las pérdidas previstas, resultando en 680 casos.

Las muestras de esputo fueron cultivadas en medio Lowenstein-Jensen por el método de Petroff modificado, en los laboratorios tipo II de la Red de Laboratorios de Tuberculosis y los cultivos positivos fueron enviados al Laboratorio de Referencia Nacional en sistema de triple embalaje de acuerdo a la norma para el transporte de sustancias infecciosas del ISP 5 .

Para la prueba de susceptibilidad a fármacos antituberculosos se empleó el método de las proporciones de Canetti, Rist y Grosset en medio Lowenstein Jensen para isoniacida, estreptomicina, etambutol y rifampicina, y el método enzimático de Wayne para estudio de pirazinamida. Cada cepa estudiada se calificó como resistente o sensible a cada una de los fármacos trabajados.

Todos los aislados que no presentaban características fenotípicas de $M$. tuberculosis fueron evaluados mediante inmunocromatografía de flujo lateral para pesquisar micobacterias no tuberculosas y descartarlas del estudio.

Los resultados concernientes al procesamiento de las cepas y a la información del paciente fueron ingresados al Sistema de Micobacterias, sistema informático con el que se emitió el informe de resultados.

\section{Resultados}

Fueron recibidos 594 casos para estudio entre junio de 2011 y julio de 2012 , lo que representa $87 \%$ de cumplimiento de la muestra calculada. En la Tabla 1 se observa el porcentaje de cumplimiento del envío de cepas por Servicio de Salud.

En las Tablas 2 y 3 se observa la resistencia inicial global y por fármaco obtenida en este estudio. Se aprecia que $91,4 \%$ de las cepas fueron sensibles y la resistencia global alcanzó a 8,6\%. De las cepas resistentes, 66,7\% lo fueron a sólo un fármaco, ya fuese a estreptomicina o isoniacida, no observándose mono-resistencia a rifampicina ni a pirazinamida. La resistencia a dos fármacos se observó en $23,5 \%$, a tres en $7,8 \%$ y a cuatro fármacos en $2 \%$.

La resistencia acumulada de cada fármaco, es decir, la resistencia total de cada medicamento, ya fuese en forma mono-resistente o poli-resistente, se muestra en la Tabla 4. Se aprecia un predominio de la resistencia a estreptomicina $(5,9 \%)$ seguida de la resistencia a isoniacida $(5,1 \%)$, rifampicina $(1,3 \%)$ y pirazinamida $(0,17 \%)$. La resistencia simultánea para isoniacida y rifampicina, es decir, MDR, fue de $1,3 \%$.

En la Tabla 5 se muestran los casos de resistencia a isoniacida y a isoniacida más rifampicina (MDR) por Servicio de Salud.
Tabla 1. Porcentaje de cumplimiento de envío de cepas de Mycobacterium tuberculosis para estudio en el Laboratorio Nacional de Referencia de Tuberculosis-ISP

\begin{tabular}{|c|c|c|c|}
\hline Servicio de Salud & Cepas solicitadas & Cepas recibidas & $\%$ Cumplimiento \\
\hline Arica & 20 & 19 & 95 \\
\hline Iquique & 40 & 40 & 100 \\
\hline Antofagasta & 20 & 19 & 95 \\
\hline Atacama & 10 & 8 & 80 \\
\hline Coquimbo & 20 & 17 & 85 \\
\hline Aconcagua & 20 & 20 & 100 \\
\hline Viña del Mar-Quillota & 20 & 15 & 75 \\
\hline Valparaíso-San Antonio & 10 & 4 & 40 \\
\hline Libertador B. O' Higgins & 40 & 26 & 65 \\
\hline Del Maule & 20 & 15 & 75 \\
\hline Nuble & 10 & 10 & 100 \\
\hline Biobío & 40 & 40 & 100 \\
\hline Concepción & 10 & 3 & 30 \\
\hline Talcahuano & 20 & 20 & 100 \\
\hline Arauco & 20 & 19 & 95 \\
\hline Araucanía Norte & 10 & 1 & 10 \\
\hline Araucanía Sur & 20 & 20 & 100 \\
\hline Valdivia & 10 & 9 & 90 \\
\hline Del Reloncaví & 20 & 20 & 100 \\
\hline Osorno & 20 & 18 & 90 \\
\hline Chiloé & 10 & 10 & 100 \\
\hline Aysén & 10 & 7 & 70 \\
\hline Magallanes & 10 & 6 & 60 \\
\hline Metropolitano Norte & 20 & 20 & 100 \\
\hline Metropolitano Central & 60 & 60 & 100 \\
\hline Metropolitano Sur & 20 & 20 & 100 \\
\hline Metropolitano Oriente & 40 & 40 & 100 \\
\hline Metropolitano Occidente & 60 & 48 & 80 \\
\hline Metropolitano Sur Oriente & 40 & 40 & 100 \\
\hline Total país & 680 & 594 & 87 \\
\hline
\end{tabular}

Tabla 2. Resistencia inicial global de Mycobacterium tuberculosis. Chile 2011-2012

\begin{tabular}{|lcc|} 
& $\mathbf{n}$ & $\%$ \\
Total cepas & 594 & 100 \\
Sensibles & 543 & 91,4 \\
Resistentes & 51 & 8,6 \\
\hline
\end{tabular}




\begin{tabular}{|c|c|c|}
\hline & $\mathbf{n}$ & $\%$ \\
\hline S & 21 & 3, \\
\hline H & 13 & 2,2 \\
\hline$R$ & 0 & 0 \\
\hline z & 0 & 0 \\
\hline E & 0 & 0 \\
\hline SH & 9 & 1, \\
\hline$H R$ & 3 & 0, \\
\hline RE & 0 & 0 \\
\hline SHE & 0 & 0 \\
\hline SHR & 4 & 0,7 \\
\hline SHRZ & 1 & 0,2 \\
\hline SHRE & 0 & 0 \\
\hline SHREZ & 0 & 0 \\
\hline Total MDR & 8 & 1, \\
\hline \multicolumn{3}{|c|}{$\begin{array}{l}\text { S: estreptomicina. } \mathrm{H} \text { : isoniacida. } \mathrm{R} \text { : rifampicina. Z: pirazinamida } \\
\text { E: etambutol. MDR: resistente a } \mathrm{H}+\mathrm{R} \text {. }\end{array}$} \\
\hline
\end{tabular}

Tabla 4. Prevalencia de resistencia de Mycobacterium tuberculosis acumulada por fármacos. Chile 2011-2012

\begin{tabular}{|ccc|} 
Fármacos & \multicolumn{2}{c|}{ Cepas resistentes } \\
& $\mathbf{n}$ & $\%$ \\
H & 30 & 5,1 \\
R & 8 & 1,3 \\
S & 35 & 5,9 \\
E & 0 & 0 \\
Z & 1 & 0,17 \\
\hline S: estreptomicina. H: isoniacida. R: rifampicina. Z: pirazinamida. \\
E: etambutol.
\end{tabular}

Tabla 5. Resistencia inicial de Mycobacterium tuberculosis a isoniacida y MDR por Servicio de Salud. Chile 2011-2012

\begin{tabular}{|c|c|c|}
\hline Servicio de Salud & Resistencia a H & MDR \\
\hline Arica & 1 & 0 \\
\hline Iquique & 1 & 1 \\
\hline Antofagasta & 0 & 1 \\
\hline Atacama & 0 & 0 \\
\hline Coquimbo & 0 & 0 \\
\hline Aconcagua & 0 & 0 \\
\hline Viña del Mar-Quillota & 0 & 0 \\
\hline Valparaíso-San Antonio & 0 & 1 \\
\hline Libertador B. O' Higgins & 0 & 0 \\
\hline Del Maule & 2 & 0 \\
\hline Ñuble & 0 & 0 \\
\hline Biobío & 1 & 0 \\
\hline Concepción & 3 & 0 \\
\hline Talcahuano & 2 & 0 \\
\hline Arauco & 0 & 0 \\
\hline Araucanía Norte & 0 & 0 \\
\hline Araucanía Sur & 0 & 0 \\
\hline Valdivia & 0 & 0 \\
\hline Del Reloncaví & 0 & 0 \\
\hline Osorno & 0 & 0 \\
\hline Chiloé & 1 & 0 \\
\hline Aysén & 1 & 0 \\
\hline Magallanes & 0 & 0 \\
\hline Metropolitano Norte & 1 & 1 \\
\hline Metropolitano Central & 3 & 0 \\
\hline Metropolitano Sur & 3 & 1 \\
\hline Metropolitano Oriente & 0 & 0 \\
\hline Metropolitano Occidente & 1 & 2 \\
\hline Metropolitano Sur oriente & 2 & 1 \\
\hline Total país & 22 & 8 \\
\hline
\end{tabular}

\section{Discusión}

La prevalencia de resistencia global en este estudio fue de 8,6\%. En los estudios anteriores al año 2005, la resistencia global alcanzaba una prevalencia de $\sim 10 \%$ y el último estudio del año 2006 arrojó una resistencia global de $11 \%{ }^{2}$. Estas cifras muestran que la resistencia a fármacos anti-tuberculosos en los enfermos nunca antes tratados no ha presentado un aumento en el país.

En relación a la TBC-MDR, ésta corresponde a 1,3\%, observándose un aumento de este perfil respecto a los estudios anteriores: $0,4 \%$ en 1997 y $0,7 \%$ en 2001 . El año 2006 el porcentaje de MDR fue cero, pero es importante hacer notar que ese año se utilizó una muestra más pequeña de la que probablemente correspondía usar según la metodología descrita, lo que podría influir en estos resultados 2 . La prevalencia de $1,3 \%$ de MDR es preocupante, no sólo porque dobla la prevalencia del año 2001 sino también porque al tener una prevalencia mayor a $1 \%$ se habría superado el límite para que Chile sea considerado país de baja incidencia en $\mathrm{MDR}^{6}$. Sin embargo, hay que considerar que debido al bajo número 
de casos, pequeños cambios en frecuencia alteran los resultados en forma importante.

En cuanto a la resistencia por fármacos, y en comparación con los estudios anteriores, se observa una disminución de la resistencia acumulada a estreptomicina desde $9,9 \%$ en el año 2006 a 5,9\% en el 2011-12 y de la asociación estreptomicina-isoniacida ( $\mathrm{SH})$ de 3 a 1,5\%. Esta gran reducción de la resistencia a estreptomicina es concordante con el hecho de que este fármaco se ha utilizado cada vez menos en el tratamiento de la TBC, ya que hasta el cambio de normativa, estaba reservado sólo para enfermos antes tratados o casos especiales, cuyo volumen alcanza sólo a $10 \%$ del total de enfermos al año en Chile? .

Por el contrario, existe un aumento de la monoresistencia a isoniacida desde $1,4 \%$ en el año 2001 a 2,2\% en el 2011-12 (0,9\% el 2006) y también de la resistencia acumulada a este fármaco, subiendo desde 4,5\% el 2001 a 5,1\% en el $2011-12(3,9 \% \text { el } 2006)^{2}$. Isoniacida es uno de los fármacos pilares en el tratamiento anti-tuberculoso, y la resistencia secundaria a este fármaco predomina cuando existe baja adherencia al tratamiento, aumentando la posibilidad de que estas cepas resistentes se transmitan a la población ${ }^{8}$.

En relación a etambutol, las cepas analizadas nuevamente no presentaron resistencia a este fármaco, al igual que en los estudios anteriores. Este resultado es esperable debido que este fármaco se utiliza en Chile hace un poco más de 20 años y siempre asociado con los demás antituberculosos, por lo que una monoterapia encubierta es menos probable.

Ninguna cepa analizada fue mono-resistente a rifampicina durante este estudio; en cambio, el año 2006 esta cifra fue de $0,3 \%$ y el 2001 fue de $0,1 \%{ }^{2}$. La resistencia acumulada a rifampicina tiene una prevalencia de $1,3 \%$ y siempre está asociada a resistencia a isoniacida, por lo tanto, toda resistencia a rifampicina correspondió a MDR.

En cuanto a la distribución de estas cepas MDR, 62,5\% ( 5 casos) se presentó en la Región Metropolitana y 25\% (2 casos) en las regiones del norte (Tarapacá y Antofagasta), las tres regiones que presentan los más altos porcentajes de TBC en inmigrantes ${ }^{9}$ y es conocido que esta población tiene un alto riesgo de presentar resistencia a los fármacos anti-tuberculosos. En Perú, por ejemplo, en un estudio realizado entre los años 2005 y 2006, la prevalencia de resistencia global fue de 23,2\% y de TBC MDR de 5,3\% ${ }^{10}$. En Colombia, el estudio realizado entre los años 2004 y 2005 determinó una prevalencia de resistencia global en casos nuevos de 11,7\%, y de TBC-MDR de 2,38

En el presente estudio no se cuenta con el antecedente de la nacionalidad de los casos de TBC resistente, antecedente que sería importante de explorar ya que el hecho de que existan estos perfiles en enfermos chilenos nunca antes tratados estaría demostrando que existe transmisión de cepas resistentes en la comunidad.

El estudio RETUBES realizado en España entre los años 2010 y 2011 observó una resistencia acumulada de 5,7\% y un porcentaje de MDR de 1,2\% entre los pacientes sin tratamiento previo, cifras muy similares a las chilenas $^{12}$. España tiene una tasa de incidencia de TBC, en todas las formas, similar a la de Chile y una distribución por grupos de riesgo muy parecida, incluyendo las mayores tasas de incidencia en inmigrantes, por lo que la comparación del perfil de resistencia con este país puede ser valiosa para comprender el fenómeno con mayor profundidad $^{13}$.

En el año 2012 se produjeron siete casos de MDR en enfermos nunca antes tratados, los cuales fueron testeados por tener factores de riesgo para TBC-MDR (de acuerdo a la normativa vigente ese año) o porque formaban parte de este estudio de resistencia inicial. Pero con los resultados de este estudio podría esperarse al menos 20 casos de MDR primaria. Este probable sub-diagnóstico de paciente con TBC-MDR se verá subsanado con la actualización de la Norma Técnica de 2014 que estipula la vigilancia de la susceptibilidad a fármacos anti-tuberculosos en forma universal $^{3}$. De hecho, en los ocho primeros meses de vigencia de la normativa (abril a diciembre de 2014) se diagnosticaron 12 casos de TB-MDR en pacientes nunca antes tratados 9 .

En conclusión, según lo observado por este estudio, en Chile existe un aumento de la resistencia a los fármacos anti-tuberculosos, especialmente a isoniacida con perfil de mono-resistencia o en combinación con rifampicina (TBC-MDR). En este contexto, la vigilancia universal de la resistencia antimicrobiana que se ha establecido a partir de 2014 es una oportunidad, no sólo para conocer el perfil de la resistencia en forma mucho más exhaustiva, sino también para realizar un tratamiento adecuado a cada paciente según la susceptibilidad a fármacos y disminuir el tiempo de exposición de la comunidad a estas cepas resistentes.

Agradecimientos. Las autoras expresan su gratitud al personal técnico de la Sección Micobacterias Virginia Casanello C, Andrea Sanhueza T. y Bárbara Cerda J. por la realización de los procesos técnicos del estudio y confección de las bases de datos. Además, nuestros agradecimientos a todo el personal de la Red Nacional de Laboratorios de Tuberculosis que con su compromiso y dedicado trabajo hicieron posible la realización del presente estudio.

\section{Resumen}

Esta publicación presenta los resultados del estudio de resistencia inicial a fármacos anti-tuberculosos de primera línea realizado entre los años 2011 y 2012 en Chile por el Laboratorio de Referencia Nacional del Instituto de 
Salud Pública, estudio que forma parte de la vigilancia de la fármaco-resistencia en tuberculosis (TBC) promovida por la Organización Mundial de la Salud. Metodología: Estudio transversal realizado mediante un muestreo por conglomerado, representativo de todo el país según recomendaciones de la Organización Mundial de la Salud. Se realizó prueba de susceptibilidad a isoniacida, rifampicina, estreptomicina, etambutol y pirazinamida a través del método de las proporciones o método enzimático de Wayne según corresponda. Resultados: Se realizó test de susceptibilidad a 594 casos de TBC, observándose una resistencia inicial global de $8,6 \%$ y una prevalencia de multi-resistencia de 1,3\%. Además destaca la caída en la resistencia a estreptomicina y el aumento de la resistencia a isoniacida, tanto en mono-resistencia como en resistencia acumulada para ambos fármacos comparada con los estudios anteriores. No se observaron casos de mono-resistencia a rifampicina. Conclusión. Se observa un aumento de la resistencia a fármacos anti-tuberculosos en Chile la que, a pesar de ser aún baja, no deja de ser preocupante. Desde el año 2014 la vigilancia de fármacoresistencia para TBC se hace en forma universal, de modo de evitar el sub-diagnóstico y realizar un tratamiento de acuerdo al perfil de susceptibilidad de cada caso.

\section{Referencias bibliográficas}

1.- World Health Organization. Global tuberculosis report 2013. 2014. http://www.who.int/tb/ publications/global_report/en/

2.- Riquelme M, Velasco M, Rodríguez L. Actualización de la resistencia a drogas antituberculosas en Chile, 2006. Rev Chil Enf Respir [Internet]. Sociedad Chilena de Enfermedades Respiratorias; 2008 Mar [citado el 26 de febrero de 2015]; 24 (1): 60-5. Disponible en: http://www.scielo. cl/scielo.php?script=sci_arttext\&pid= S0717-73482008000100012\&lng= es\&nrm=iso\&tlng=es

3.- Programa Nacional de Control y Eliminación de la Tuberculosis. Normas técnicas para el control y la eliminación de la tuberculosis [Internet]. 2014. Disponible en: http://web.minsal.cl/ sites/default/files/NORMA_TECNICA TUBERCULOSIS.pdf

4.- World Health Organization. Strategic approach for the strengthening of laboratory services for tuberculosis control. 2009. http://whqlibdoc.
who.int/hq/2006/WHO_HTM_TB_2006.364_ eng.pdf

5.- Instituto de Salud Pública. Norma técnica para el transporte de sustancias infecciosas a nivel nacional hacia el Instituto de Salud Pública (ISP) 2008 [Internet]. 2008. Disponible en: http://www.ispch.cl/sites/default/files/ documento/2013/03/normativa02.pdf

6.- Piffardi S, Valenzuela P, Velasco M, Lepe R, Vergara M, Zúñiga M. Resistencia inicial a drogas antituberculosas: Chile, 1997. Enfermedades respir cir torac 1999; 15 (1): 9-17.

7.- Programa Nacional de Control y Eliminación de la Tuberculosis. Manual de Organización y Normas Técnicas. Ministerio de Salud. 2005. http://web.minsal.cl/portal/url/item/803048171a cc60f8e04001011f0148e2.pdf

8.- De la Iglesia A, Morbidoni H. Mecanismos de acción y de resistencia a rifampicina e isoniacida en Mycobacterium tuberculosis: nueva información sobre viejos conocidos. Rev Argent microbiol 2006; 38: 97-109.

9.- Programa Nacional de Control y Eliminación de la Tuberculosis. Informe de la situación de la tuberculosis en Chile 2014. Ministerio de Salud. 2015. http://web.minsal.cl/portal/url/item/80304 8171 acc60f8e04001011f0148e2.pdf

10.- Asencios L, Quispe N, Mendoza-Ticona A, Leo E, Jave O. Vigilancia nacional de la resistencia a medicamentos. Rev Perú Med Exp Salud Pública 2009; 26 (3): 278-87.

11.- Garzón M, Angée D, Llerena C, Orjuela D, Victoria J. Vigilancia de la resistencia del Mycobacterium tuberculosis a los fármacos antituberculosos, Colombia 2004-2005. ev Biomédica 2008; 28: 319-26.

12.- Blanquer R, Rodrigo T, Casals M, Manzano J, García J, Carpe J, et al. Resistencia a fármacos antituberculosos de primera línea en España durante 2010-2011. Estudio RETUBES. Arch bronconeumol 2014; 51 (1): 24-30.

13.- Centro Nacional de Epidemiología. Informe epidemiológico sobre la situación de la tuberculosis en España. Instituto de Salud Carlos III [Internet]. 2013. Disponible en: http://www.isciii.es/ISCIII/es/contenidos/ fd-servicios-cientifico-tecnicos/fd-vigilanciasalertas/fd-enfermedades/TB_Informe_2013 CNE_9febrero2015.pdf 